\title{
Supportive care for patients with brain metastases from lung cancer
}

\author{
Akanksha Sharma ${ }^{1}$ Maciej M. Mrugala ${ }^{2}$ \\ ${ }^{1}$ Department of Translational Neurosciences and Neurotherapeutics; John Wayne Cancer Institute and Pacific Neuroscience Institute, Santa Monica, \\ CA, USA; ${ }^{2}$ Department of Neurology, Comprehensive Neuro-Oncology Program, Mayo Clinic Cancer Center, Phoenix, AZ, USA \\ Contributions: (I) Conception and design: Both authors; (II) Administrative support: None; (III) Provision of study materials or patients: Both authors; \\ (IV) Collection and assembly of data: Both authors; (V) Data analysis and interpretation: Both authors; (VI) Manuscript writing: Both authors; \\ (VII) Final approval of manuscript: Both authors. \\ Correspondence to: Maciej M. Mrugala. Comprehensive Neuro-Oncology Program, Mayo Clinic Cancer Center, Phoenix, AZ, USA. \\ Email: mrugala.maciej@mayo.edu.
}

\begin{abstract}
Lung cancer is the most common cause of intracranial metastases (ICM). Metastases in the brain can result in a broad range of uncomfortable symptoms and significant morbidity secondary to neurological disability. Treatment options can range from surgical resection of solitary metastases to radiotherapy and more recently systemic targeted therapies and immunotherapy. Patient survival continues to improve with innovations made in treatments for this condition, but each of these treatments carry their own adverse effects that must be appropriately managed. These patients can benefit greatly from multidisciplinary care throughout the course of their disease. Clinicians involved in their care must be equipped with the ability to communicate skillfully and compassionately and set expectations for the road ahead, including symptoms, treatment plans, and prognosis. Involvement of a palliative care team can be very helpful, especially for patients who are nearing the terminal stages of the disease. Palliative care skills may be invaluable in the management of symptoms and can ease suffering for patients and their caregivers, thus allowing for maximum quality of life for as long as possible. End of life may bring its own complications and challenges; and opinion of an experienced and knowledgeable clinician can alleviate the pain and distress of the patient and also bring peace to the caregivers and loved ones.
\end{abstract}

Keywords: Brain metastasis; lung cancer; supportive care

Submitted Feb 18, 2020. Accepted for publication Sep 29, 2020.

doi: $10.21037 /$ jtd-2019-rbmlc-11

View this article at: http://dx.doi.org/10.21037/jtd-2019-rbmlc-11

\section{Introduction}

The incidence of intracranial metastases (ICM) is noted to be the highest in the lung cancer population. Rates for metastases to the central nervous system (CNS) can be as high as $20 \%$ (1). The incidence of brain metastases appears to have increased over time as treatments for the systemic disease have improved, resulting in longer survival and thus increased time to develop ICM (2).

ICM management in lung cancer has traditionally involved a combination of surgical resection for isolated and/or symptomatic lesions, combined with radiation therapy. Whole brain radiation therapy (WBRT) was a mainstay for management of ICM, but several randomized controlled trials in the last two decades have compared stereotactic radiosurgery (SRS) with WBRT and concluded that the former can result in similar survival outcomes with less neurotoxicity, thus resulting in SRS increasingly being used over WBRT (3-6). In recent years, the use of immunotherapy for tumors with appropriate genetic mutations has also become more prevalent given excellent intracranial efficacy of many of these agents resulting in remarkable progression-free and overall survival $(7,8)$. 


\section{Surgical treatment}

Surgical resection of lung cancer related ICM is usually recommended in certain situations. Often there may be a need for a tissue diagnosis, and biopsy or resection of a single lesion is undertaken if reasonable and safe. More often, lesions that are causing mass effect or edema with secondary clinical symptoms such as impending hydrocephalus or herniation may need to be surgically removed. Patients with solitary lesions and lesions too large for radiation therapy (generally $>3 \mathrm{~cm}$ ) are also favorable surgical candidates when a surgically accessible location can be identified. In fact, oligometastatic disease in non-small cell lung cancer can often be removed with curative intent (9). Radiation failure or radiation necrosis, hemorrhage into the lesion, or large symptomatic cystic lesions may be other indications for surgical intervention of ICM (10). Patients receiving surgical intervention for ICM will, in most cases, experience a resolution of neurologic symptoms and will improve post-operatively. When not performed for clinical reasons, outcomes depend on the location of the lesion and the pre-operative functional status of the patient. It is important to consider potential neurological outcomes depending on the location of the lesion, and to closely follow neurological exam post-operatively. Generally, patients can be discharged soon after surgery with appropriate therapies (physical and occupational therapy) in place if needed, and close outpatient follow up is recommended. In rare cases, clinical course may be complicated by post-operative hemorrhage at the site of the resection or an infection resulting in additional morbidity.

Steroids are an important consideration with surgical intervention. Most patients who undergo surgery may already be on steroids (since edema/mass effect may be prompting the intervention) or are placed on steroids right before the procedure and on a taper post-operatively. Steroids may reduce the local edema and inflammation which can be helpful for the surgery. In addition, continued use post-operatively can continue to reduce the inflammatory environment and reduce risk for seizures and other complications. Dexamethasone is generally the steroid of choice given longer half-life (up to 36 hours) and low mineralocorticoid activity (11). However, a clear taper plan must be in place for all patients being discharged from the hospital on steroids. No evidence based taper plan recommendation exists at this time. In most institutions, dexamethasone is reduced by $2 \mathrm{mg}$ every 3-7 days until complete discontinuation. Dexamethasone should be dosed either as a larger, cumulative dose in the AM only or divided between AM and afternoon, to reduce its effect on the quality and duration of sleep and resulting downstream side effects.

Patients with an ICM who have had any episode concerning for a seizure should be started on an antiepileptic drug (AED) since the risk of subsequent seizures is high in this population. There is not enough evidence to guide how long AEDs should be continued postoperatively and how/when a patient can be tapered off completely, if ever. Prophylactic AEDs for patients with ICM without any epileptic seizures at all is an area of continued controversy. Several reviews and consensus guidelines recommend against prophylactic initiation in these patients given the balance of side effects versus benefit (12). On the other hand, there is concern that the studies these guidelines were based on did not use newer, AEDs. It remains a common practice to start AEDs in patients with brain metastases pre- and post-operatively. The drug that is most commonly used in this setting is levetiracetam, which has comparatively fewer major side effects and minimal drug-to-drug interactions. In cases where the antiepileptic drug was used prophylactically without clear evidence of seizure, it should be weaned off as soon as possible post-operatively.

\section{Radiotherapy}

The decision to use SRS versus WBRT involves consideration of many variables and factors. Radiation therapy to the brain for metastatic disease from any type of cancer is associated with a number of toxicities that requires appropriate management. Not every patient will demonstrate toxicity. Many factors, including age, existing comorbidities, functional status, radiation dose and size and location of lesion treated may impact the side effects experienced by an individual patient.

\section{Acute radiation toxicity}

These symptoms usually start between several hours to 2 weeks out from the treatment. Patients may experience headache, vomiting, drowsiness and lethargy, and worsening of pre-existing neurological deficits. There may also be a component of encephalopathy or confusion in this period. Depending on the size and location of the lesion, significant mass effect and edema leading to herniation and death has been reported $(13,14)$. 


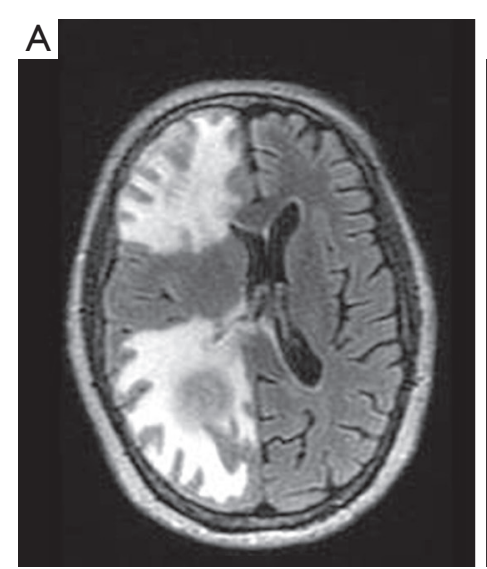

6 months after SRS (steroids started)

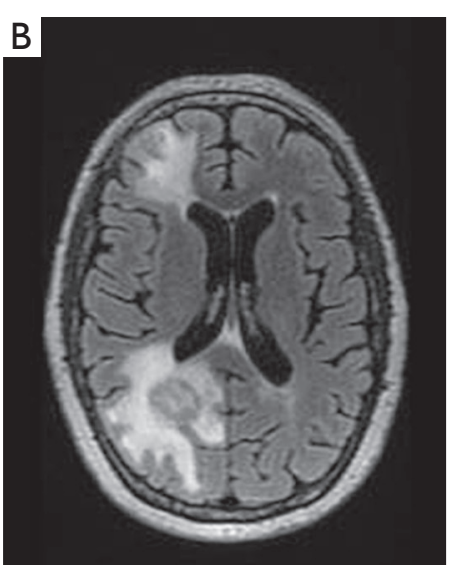

9 months after SRS (no steroids)

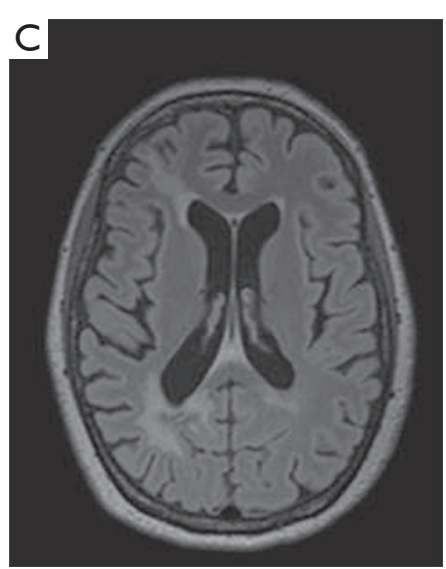

15 months after SRS (no steroids)

Figure 1 MRI of brain, axial T2 FLAIR sequences. Early delayed radiation toxicity in a 73-year-old woman with metastatic non-small cell lung cancer. Patient developed several brain metastases and was treated with stereotactic radiosurgery (SRS). Six months after treatment the patient was admitted to the hospital with increased headaches, left leg weakness, and falls. MRI identified increased vasogenic edema around the treated metastases (A). She was started on steroids and improved clinically. Three months later, MRI showed further improvement of vasogenic edema off of steroids, and patient was clinically stable (B). Fifteen months after SRS patient remains neurologically stable, off steroids with further radiographic improvement (C). Clinical and radiographic course is consistent with treatment effect rather than disease progression (early delayed radiation toxicity). SRS, stereotactic radiosurgery.

\section{Management}

Steroids are the recommended treatment to prevent and/ or treat any acute symptoms. In cases where there is preexisting edema around the lesion, the lesion is large, or where the lesion(s) is located in a vulnerable location (i.e., posterior fossa or spinal cord), it may be prudent to start steroids 48-72 hours prior to starting the treatment, and slowly taper them off over a couple weeks. Doses recommended range from 4 to $16 \mathrm{mg}$ of dexamethasone daily in divided or cumulative doses, ideally before late afternoon as to minimize negative effects on sleep. When symptoms start soon after radiation, steroids can be started immediately at an appropriate dosage and should be tapered off over a few weeks. As discussed in the previous section, no evidence-based taper regimen exists for steroids in this scenario. In our practice we usually recommend decreasing the dose by $2 \mathrm{mg}$ every $1-2$ weeks depending on the patient's clinical symptoms and tolerance.

\section{Early delayed symptoms}

This is the term usually used for symptoms that occur in the 2 -week to 6 -month window after radiation treatment. Several of the symptoms are similar to the acute symptoms-headache, fatigue, lethargy, and worsening of pre-existing neurological focal deficits. With the neurological decline there can be concern of tumor progression. Imaging does not always prove to be helpful in these cases. There can be changes seen after radiation with both primary and secondary brain tumors that are similar to what is seen with progression. This makes it difficult to distinguish between true progression and "pseudoprogression," especially when there are clinical symptoms. Concomitant use of immunotherapy can increase the risk for "pseudoprogression" and careful review of patient's systemic therapy may be helpful in interpretation of the imaging and clinical management decision making. Transient cognitive impairment has also been seen in many cases in this time period.

\section{Management}

Steroids are the treatment of choice for these symptoms, generally with a slower taper and lower doses than with the acute, more urgent syndromes. Usually starting with doses of $8 \mathrm{mg}$ daily and slowly tapering over a period of weeks may be appropriate. Time and close surveillance with repeat imaging is often an important strategy in these cases, to determine if the radiographic changes are truly due to progression or are a result of treatment effect. Figure 1 illustrates a case of early delayed radiation toxicity where 
there was concern raised for tumor progression.

The use of steroids may dampen the activity and effect of the immunotherapy which is not ideal for the patient in the long run. Thus, steroids should only be started in situations where there is edema significant enough to cause clinical symptoms (such as weakness, seizures, encephalopathy, etc.) with corresponding radiographic changes, and no other reasonable explanation. If there is no or only minimal response to the steroids, or risk of immunotherapy compromise is greater than the benefit that will be gained from the steroids, alternate treatment options (such as bevacizumab, discussed below), should be considered.

\section{Late delayed symptoms}

After more than 6 months from treatment have passed, delayed complications can arise and typically include increased risk for focal radiation necrosis and cognitive impairment with leukoencephalopathy. There is no clear "end period" to this window_changes may be seen even decades after radiation therapy. As survival from lung cancer improves, we are seeing increasing rates of necrosis and delayed symptoms from radiation therapy in our patients with brain metastases (15). Focal radiation necrosis can appear as a contrast-enhancing lesion around the area of treatment and often raises concern for recurrence. Risk factors for radiation necrosis continue to be explored, but there appears to be a higher risk with larger lesions, higher radiation doses, repeated treatment, surgical resection and tumors with certain genetic markers that lead to longer survival $(16,17)$. Radiation necrosis has been reported to be seen as far as 5 years out from treatment though this is somewhat unusual-the majority of cases occur between 6 months to 2 years from treatment $(15,18)$. Patients may present with recurrent neurological deficits (mimicking the original lesion), seizures, headaches, lethargy, personality changes, etc.; these changes can present acutely or gradually.

Cognitive dysfunction in these cases can range from mild to profound impairment. This may be much worse in cases of WBRT, but it greatly depends on the individual patient and their cognitive tolerance. More research is needed in this area as currently it is not possible to predict who will develop cognitive changes and to what degree. Often imaging will demonstrate periventricular or perilesional white matter changes consistent with leukoencephalopathy. Severe dementia from radiation therapy is quite rare but does occur and can be devastating.

\section{Management}

Radiation necrosis can be spontaneously reversible at times, resolving on follow up imaging without intervention. In other cases, steroids may need to be used.

Bevacizumab, a vascular endothelial growth factor inhibitor, is recommended as a second line treatment in this setting and can be very helpful in reducing the inflammation/edema associated with radiation necrosis, resulting in clinical improvement $(19,20)$. Generally, a dosage of $7.5 \mathrm{mg} / \mathrm{kg}$ every 3 weeks is used, with re-imaging and clinical reassessment after 2-3 doses. Patients may require as few as 2-3 doses, but some require several months of treatment before clinical and radiographic improvement can be demonstrated (20). In our practice, we aim for both clinical and radiographic improvement prior to holding bevacizumab. The drug can be used for a re-challenge later should the necrosis re-present itself.

There is unfortunately no proven treatment for the cognitive dysfunction or dementia seen in these patients. Various medications used for the treatment of dementia such as donepezil may be tried but benefit is unclear in this setting (21). Methylphenidate or modafinil may also be helpful in these patients to varying degrees $(21,22)$. Methylphenidate may improve concentration and focus. Generally patients are started on $5 \mathrm{mg}$ daily in the morning, with a second dose usually added mid-morning or early afternoon. If tolerated without excessive anxiety, hypertension, tachycardia, or gastrointestinal side effects, dosage can be uptitrated to $15 \mathrm{mg}$ twice daily if the patient appears to be benefiting. Modafinil helps more with alertness, and is typically started at $100 \mathrm{mg}$ daily and can be uptitrated to $200 \mathrm{mg}$, usually with AM dosing.

Results of a double blind, placebo-controlled trial by Brown et al. published in 2013 (RTOG 0614) found that the use of memantine (20 $\mathrm{mg}$ per day) during WBRT delayed time to cognitive decline and the drug appeared to significantly reduce the rate of decline in executive and processing function for patients who were receiving WBRT (23). This study has resulted in the incorporation of memantine in most centers when WBRT is being used, though the dosing may be limited by toxicity (side effects can include GI upset). Another strategy used to reduce harm is to design plans that avoid the hippocampus-results from a phase III study (NRG CC-001) that combined hippocampal avoidance and memantine were recently reported and found that cognitive function was better preserved, and patients in the intervention group reported less fatigue and less difficulty with speech and memory (24). 
Table 1 Common side effects associated with targeted therapy and immunotherapy in patients with metastatic lung cancer

\begin{tabular}{|c|c|c|}
\hline Class of drug & Examples of drugs in group & Reported toxicities \\
\hline PDL-1 inhibitors ${ }^{*},{ }^{* *}$ & $\begin{array}{l}\text { Atezolizumab, avelumab, } \\
\text { durvalumab }\end{array}$ & $\begin{array}{l}\text { Immune related myositis, adrenal insufficiency, diabetes mellitus, cardiovascular toxicity, } \\
\text { immune mediated colitis, hepatotoxicity, hypophysitis, infections, uveitis, pulmonary } \\
\text { toxicity, thyroid disorders, immune mediated neurological disorders including } \\
\text { demyelinating disorders, myositis, encephalitis. Possible increased risk of radiation } \\
\text { necrosis (pseudoprogression) }\end{array}$ \\
\hline
\end{tabular}

*note that endocrinopathies from PD-1 and PDL-1 inhibitors may frequently lead to significant fatigue and can exacerbate pre-existing neurological symptoms in ICM patients. ${ }^{* *}$ the risk of pseudoprogression is higher when immune therapy is combined with radiation. ICM, intracranial metastases.

\section{Systemic treatment}

Small-cell lung cancer with brain metastases (extensive disease) may be systemically treated with a platinum and etoposide combination, and in the last two years there has been a recommendation to add atezolizumab to this combination in the upfront setting (25). Platinum-based therapies are known primarily for contributing towards bone marrow suppression, nausea and vomiting, fatigue, weakness, electrolyte abnormalities and peripheral neuropathy. Similar side effects are seen with etoposide, though it has a lower rate of peripheral neuropathy but increased rate of alopecia which can be distressing to the patient. Atezolizumab may cause weakness, fatigue, myalgias, gastrointestinal distress and cytopenias. There is also a concern for severe immunemediated myositis which has been reported in recent months (26). Patients on this regimen should be educated on reporting their symptoms early and regularly, since dose adjustments or discontinuation may be necessary.

In recent years, treatment of non-small lung cancer has changed dramatically especially when an oncogenic driver can be identified. In these cases, even if brain metastases are identified at initial diagnosis, first-line therapy may be a targeted agent. There is a broad range of these agents now available, depending on the gene translocation or mutation identified. Many of these drugs are still early stages of clinical use, and long-term impact and rare side effects are still being observed and explored. Some of the common adverse effects associated with these agents are presented in Table 1. Prescribing clinicians are advised to review carefully and discuss with their patient the expected side effect profile of the drug they are using, and encourage open channels of communication for any new or unusual symptoms that may arise. As with other treatments, tolerance to these drugs is highly variable in the patient population, with many known and unknown factors at play. It is not yet clear which risk factors may predispose a patient to a higher risk of developing side effects with these drugs. Intracranial efficacy of these drugs is quite high providing very good disease control. There is concern, however, that there might be an increased immune reaction around lesions, especially when combined with radiation. Current recommendations suggest frequent follow up imaging and use of the Response Assessment in Neuro-Oncology for immunotherapy (iRANO) criteria before making any decisions regarding progression unless the patient is clinically worse (27).

\section{End of life}

Median survival in lung cancer patients with brain metastases continues to lengthen secondary to the significant advances made in recent decades, but it remains a severely life-limiting condition. Generally, this phase 
is reached when there are no longer any viable surgical, radiation or systemic options available, no experimental or clinical trial options exist, or if the patient's functional status is too poor to continue any type of treatment. With brain metastases, neurological and cognitive impairment is an expected and prominent part of the eventual decline. It may be possible to both continue treatment and maintain optimism and hope for extended survival but also have realistic, practical discussions on goals of care and advanced directives. The responsibility to discuss end of life issues often falls on the oncologist as the primary treating provider, but may be shared with the palliative care provider or the primary care provider. A large body of literature now demonstrates the benefit of early palliative care in both quality and quantity of life for patients with metastatic cancer, especially lung cancer $(28,29)$. Anticipating the decline of the patient based on clinical experience and preparing the patient and caregiver/family for the coming stages allows for the best possible care.

\section{Trajectory of decline}

Clinical status and function of a patient with replace with ICM depends largely on the location of the lesions, symptom burden, and how the patient tolerates treatment. Often, the patient may be completely asymptomatic, especially after treatment with steroids or when on a targeted agent. The course for patient with ICM is therefore not one of stepwise decline, but one where they be clinically stable for a long period of time, and then acutely decline. These acute triggers may be infections, exacerbations of comorbidities, tumor progression, lack of response to therapy, side effects to therapy, new neurological deficits, etc. Patients may recover from the acute decline but not return to their previous baseline, and then either continue to move on a downwards trajectory or stay steady again until the next setback.

\section{Recommending transition in treatment goals}

It can be a challenge for providers who have been involved in the care of a patient for a prolonged period of time to anticipate and realize that the patient is now declining and entering the final stages of their disease process. There is an important role here for the oncologist as the primary provider, whom the patient and family trusts, to provide guidance and advice. It is helpful to evaluate the patient's and their caregiver's goals at regular intervals, or when the "triggers of decline" occur. Table 2 highlights some of these possible "triggers" that the oncologist or other primary providers may keep in mind as possible reminders to encourage these conversations with the patient and caregivers.

Several questions are important to address in these conversations. Are we still focused on quantity of life, regardless of the quality of that of life (excluding provider's own bias of what quality of life should be)? Are we interested only in measures focused on comfort, or are we accepting of a certain level of discomfort if it means more time with family? It is important to conduct these conversations with no prior biases or answers in mind, since responses will be highly individualized and dependent on the patient and family, their goals, culture, understanding of the condition, and personal beliefs. These responses can be helpful in creating a recommendation. If the patient indicates that they cannot bear the thought of further radiation or tolerate side effects from immunotherapy, and would rather spend their time at home with family or at a favorite location, perhaps it may be worthwhile to discuss how we can change our efforts towards fulfilling those goals instead. In this context, it is not helpful to use the terms "stopping all treatment" and "doing everything or nothing." Instead, we are simply changing the direction and path of care and the eventual goals of the care, but still providing care and treatment. Patients and their families will not be abandoned at this juncture simply because they are choosing a different goal, and thus a different treatment, to achieve that goal.

\section{Symptoms towards the end of life}

Fatigue and mild confusion may be the initial manifestations of decline, with or without focal neurological deficits. As the condition progresses, the confusion gets worse and fatigue and somnolence becomes prominent. Patients may spend more time in bed, sleeping, and less time alert and awake participating in the world. They may complain of headaches, blurring of vision, and trouble with swallowing or speaking. There may be trouble with executive functioning and completing simple tasks. Focal weakness can further the disability, and increase the time the patient spends in bed. Bodily pain may occur depending on the extent of the systemic disease, or secondary to immobility resulting from neurological decline. Seizures may occur in a small percentage of patients as a new symptom in the last month of life (30). Dysphagia can result in decreased oral intake, and subsequent malnutrition, dehydration, inability to take medications orally, and electrolyte imbalances that 
Table 2 Triggers of decline for patients with ICM

New imaging demonstrating worsening radiation necrosis or progressive disease
New line of chemotherapy or other treatment
Difficulty with language or speech
Difficulty with swallowing
New weakness or clumsiness
New cognitive difficulty or worsening cognitive issues
Personality changes (sudden outbursts of anger, or are they becoming irritable, or withdrawn and apathetic, or perhaps inappropriate and
disinhibited?)
Fall resulting in serious injury (fracture, etc.)
Caregiver fatigue and distress
Uncontrolled symptoms (especially neurological in nature)
Patient and caregiver request
Steroid dependence
Significant drop in performance status

ICM, intracranial metastases.

further contribute to lethargy and eventual comatose state. Patients may aspirate and develop pneumonia and resultant dyspnea. Thromboses and pulmonary embolisms may occur. Urinary tract or skin infections may develop. Generally, death is secondary to the combination of factors-lack of food and fluid intake, infection, and possible embolism.

\section{Management considerations towards the end of life}

It is difficult to overstate the importance of considering the vast differences of opinion when it comes to palliative care, comfort care and hospice care not only of patients but of clinicians also. A growing body of research demonstrates that it may be best to inquire honestly and genuinely into a patient and family's personal preferences to tailor care at this stage of life. For example, in some cultures and religions, it would be unacceptable to change code status to "do not resuscitate" but the family may still want an approach that focuses on comfort without the term of "hospice," which carries the stigma of futility.

If hospice care is acceptable and available to the patient and family, it can improve both the quality and quantity of life and can be a significant source of support to the patient's family. Hospice resources, however, will vary greatly depending on the needs of the patient, the medical system, and the culture. Preparing the patient for this transition is critically important, as this can be a point where the patient may feel abandoned. Oncologists can still remain very involved in the care, depending on the system, and may be especially helpful since ICM patients can experience some challenging symptoms not all hospice providers may be comfortable with (seizures, or intracranial edema). Different symptoms that can be expected at the end of life, as well as suggestions on management are provided as Table 3.

\section{Nutrition}

Nutrition is frequently a challenging subject to discuss and can trigger strong emotions in the patient and the family. Not only do families feel guilt and grief at the thought of potentially "starving" their loved ones; patients themselves may feel fearful at the thought of not being able to eat. In some cultures and religions, this can be especially crucialfood may hold more meaning than just a means for nutrition. Nutrition in terminally and chronically ill patients has not shown clinical benefit, and in cancer patients the palliative use of nutrition support is rarely indicated, especially not towards the end of life (31). Towards the end of life, as organs begin to shut down, electrolyte imbalances result in increased nausea and changes in taste and appetite, and often abdominal bloating and fluid retention, resulting in less hunger and desire to eat. In this phase, forcing food or drink 
Table 3 Management of symptoms and needs at the end of life in patients with metastatic brain cancer

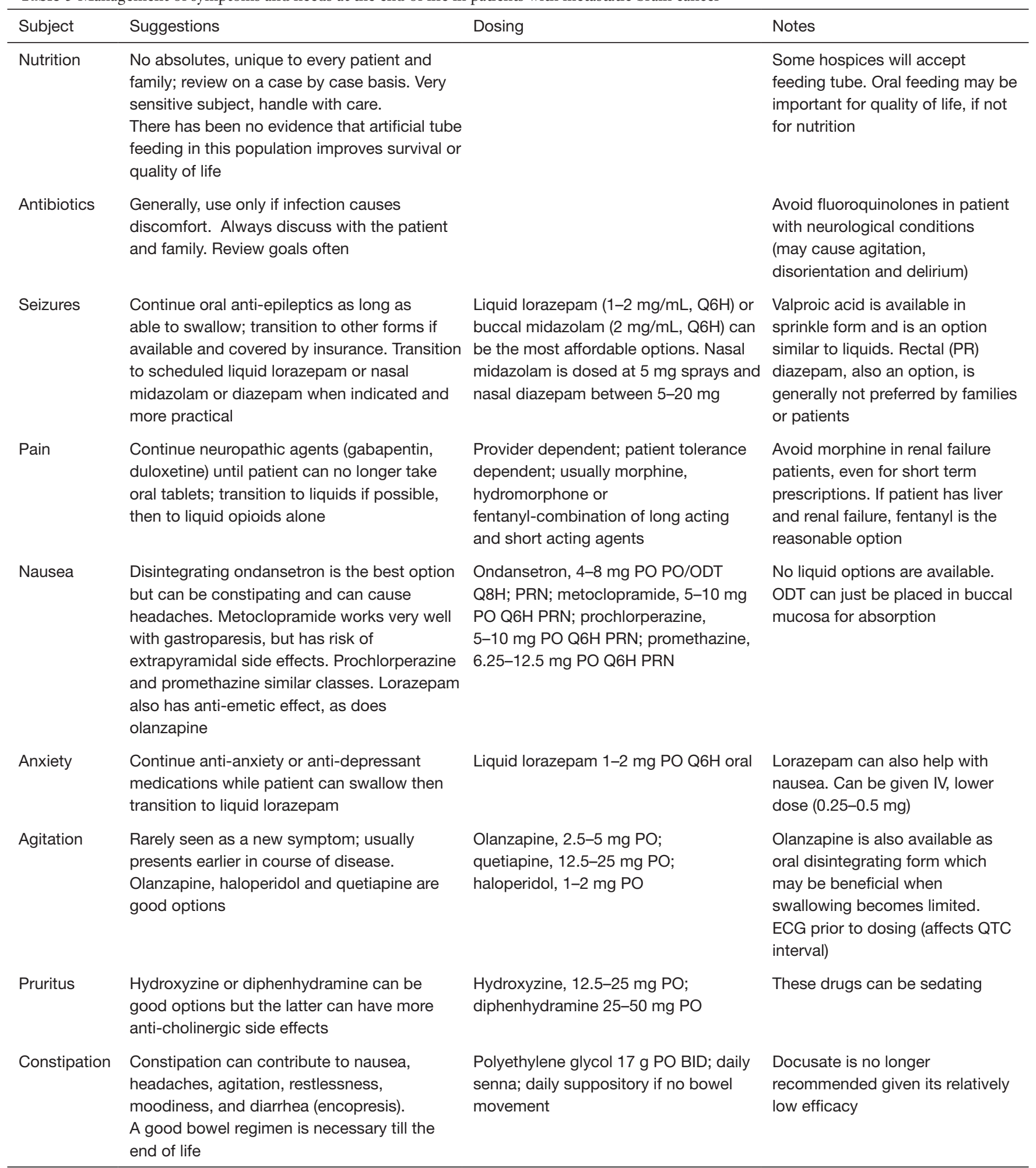

PO, by mouth; PR, per rectum. 
may actually be more uncomfortable for the patient, and it will not have intended nutritional benefits. Artificial feeding at this stage will also not change the course of the disease, and thus will not change the outcome, but may only increase the discomfort for the patient. Each case has to be reviewed on a case by case basis and palliative care and hospice assistance can be very helpful in these situations.

\section{Antibiotics}

Similar to nutrition, the use of antibiotics to treat infection can be an emotional and challenging subject to discuss. If the goal is comfort, however, and if the treatment is unlikely to change the eventual course, it may be worthwhile to only treat infections that cause discomfort or only use agents that can treat the discomfort itself (for example, using phenazopyridine with or without antibiotic for a urinary tract infection towards the end of life).

\section{Seizures}

Generally, patients with ICM have already had a seizure and are on AEDs. A small percentage may develop seizures at the end of life. A critical problem that complicates management in this patient population is dysphagia. Many AEDs are oral and missing doses due to dysphagia may pose a challenge and increase the risk of seizures-a complication to be avoided in the comfort care/hospice setting. It is important to prepare families for this phase and also have a plan in place well in advance. Alternate forms of the medications may need to be considered, or the patient may need to be switched to a benzodiazepine regimen. There are tablet, liquid, buccal, rectal, intramuscular, intravenous and subcutaneous formulations of benzodiazepines that can be used depending on availability in the country and insurance coverage. Most often, buccal lorazepam or midazolam may be the easiest option, and can be dosed even in a lethargic patient. Intranasal midazolam is also an efficient and comfortable option when affordable for use. Rectal options are less popular at this point in life due to the family's discomfort and concern for dignity. Often, patients may have ports or central lines in place and intravenous benzodiazepines can be used in these cases, or hospice staff can start subcutaneous infusions.

\section{Pain}

The level of pain in this population of patients will depend on the combination of the systemic and intracranial disease burden. Opioid use at the end of life is common in the metastatic cancer population. The type of opiate, dosage and route depends on the location of the patient, access, patient's alertness and ability to swallow. Generally, a low level of continuous pain medication may be provided through a patch or an intravenous infusion, with breakthrough pain medication available orally or through intravenous options.

\section{Anxiety/agitation}

Towards the final stages of life, benzodiazepines may be helpful for anxiety, existential distress, and the discomfort that comes with dyspnea and air hunger. These can be dosed orally, buccally, rectally, or nasally, and given as intramuscular/intravenous injections. Options include lorazepam, clonazepam, diazepam and midazolam. Haldol may be helpful for agitation or restlessness. Hydroxyzine is another option, and has the added benefit of treating pruritus, which can present itself with the organ dysfunction which is occurring at end of life (secondary to uremia and hepatic failure).

\section{Nausea}

Nausea is another prevalent symptom at this stage. Treatment may include anti-emetics-metoclopramide may be especially helpful since it can treat the gastroparesis frequently encountered at this stage. Ondansetron and olanzapine are the only options available in disintegrating forms that can be placed on the buccal mucosa. Ondansetron may contribute to constipation, however, and also to headaches. Lorazepam can be as anti-emetic use and is available in liquid form. Olanzapine and haloperidol can also be helpful, but can be very sedating.

\section{Constipation}

It is important to address constipation, especially with opioids on board. Constipation can be quite distressing and uncomfortable at the end of life. It is frequently underappreciated given the misconception that with very little intake there should be little output. There is also a hesitation given the fact the patients are bedbound at this point. However, a bowel regimen can be very helpful in preventing additional distress to the patient. Suppositories and enemas are also available and families should be 
informed that these may need to be used if several days have gone by without a bowel movement, even in a patient with poor $\mathrm{PO}$ intake.

\section{Conclusions}

The holistic management of the patient with ICM from lung cancer requires multidisciplinary care and close engagement of the primary care team from diagnosis to death. Frequent assessments along with clinical evaluation and diagnostic testing can help identify problems promptly and address them in a timely manner. Different symptoms will arise at different stages of the disease, depending on the treatment course chosen. Open communication between the healthcare provider and the patient and family will decrease anxiety related to unexpected symptoms and prognosis. Many treatment options exist to manage symptoms at different stages of metastatic brain disease. Balancing the benefits of these treatments with potential side effects must be taken into consideration. Realistic goal setting is important and these goals should be frequently reassessed as they may change over time as the disease progresses. To avoid a sense of abandonment, clear communication about transition to hospice and involvement of the primary medical team should take place. Optimal management of this patient population may frequently be challenging for the healthcare provider and a high degree of sensitivity and engagement is necessary to offer the best quality of life for the patient.

\section{Acknowledgments}

Funding: None.

\section{Footnote}

Provenance and Peer Review: This article was commissioned by the Guest Editor (Lucyna Kepka) for the series "Radiotherapy for Brain Metastases from Lung Cancer" published in Fournal of Thoracic Disease. The article has undergone external peer review.

Conflicts of Interest: Both authors have completed the ICMJE uniform disclosure form (available at http://dx.doi. org/10.21037/jtd-2019-rbmlc-11). The series "Radiotherapy for Brain Metastases from Lung Cancer" was commissioned by the editorial office without any funding or sponsorship. The authors have no other conflicts of interest to declare.
Ethical Statement: The authors are accountable for all aspects of the work in ensuring that questions related to the accuracy or integrity of any part of the work are appropriately investigated and resolved.

Open Access Statement: This is an Open Access article distributed in accordance with the Creative Commons Attribution-NonCommercial-NoDerivs 4.0 International License (CC BY-NC-ND 4.0), which permits the noncommercial replication and distribution of the article with the strict proviso that no changes or edits are made and the original work is properly cited (including links to both the formal publication through the relevant DOI and the license). See: https://creativecommons.org/licenses/by-nc-nd/4.0/.

\section{References}

1. Barnholtz-Sloan JS, Sloan AE, Davis FG, et al. Incidence proportions of brain metastases in patients diagnosed (1973 to 2001) in the Metropolitan Detroit Cancer Surveillance System. J Clin Oncol 2004;22:2865-72.

2. Herbst RS, Morgensztern D, Boshoff C. The biology and management of non-small cell lung cancer. Nature 2018;553:446-54.

3. Brown PD, Jaeckle K, Ballman KV, et al. Effect of Radiosurgery Alone vs Radiosurgery With Whole Brain Radiation Therapy on Cognitive Function in Patients With 1 to 3 Brain Metastases: A Randomized Clinical Trial. JAMA 2016;316:401-9.

4. Brown PD, Ballman KV, Cerhan JH, et al. Postoperative stereotactic radiosurgery compared with whole brain radiotherapy for resected metastatic brain disease (NCCTG N107C/CEC.3): a multicentre, randomised, controlled, phase 3 trial. Lancet Oncol 2017;18:1049-60.

5. Yamamoto M, Serizawa T, Higuchi $Y$, et al. A Multiinstitutional Prospective Observational Study of Stereotactic Radiosurgery for Patients With Multiple Brain Metastases (JLGK0901 Study Update): Irradiationrelated Complications and Long-term Maintenance of Mini-Mental State Examination Scores. Int J Radiat Oncol Biol Phys 2017;99:31-40.

6. Aoyama H, Shirato H, Tago M, et al. Stereotactic radiosurgery plus whole-brain radiation therapy vs stereotactic radiosurgery alone for treatment of brain metastases: a randomized controlled trial. JAMA 2006;295:2483-91.

7. Shaw AT, Gandhi L, Gadgeel S, et al. Alectinib in ALKpositive, crizotinib-resistant, non-small-cell lung cancer: 
a single-group, multicentre, phase 2 trial. Lancet Oncol 2016;17:234-42.

8. Soria JC, Ohe Y, Vansteenkiste J, et al. Osimertinib in Untreated EGFR-Mutated Advanced Non-Small-Cell Lung Cancer. N Engl J Med 2018;378:113-25.

9. Li D, Zhu X, Wang H, et al. Should aggressive thoracic therapy be performed in patients with synchronous oligometastatic non-small cell lung cancer? A metaanalysis. J Thorac Dis 2017;9:310-7.

10. Ewend MG, Morris DE, Carey LA, et al. Guidelines for the initial management of metastatic brain tumors: role of surgery, radiosurgery, and radiation therapy. J Natl Compr Canc Netw 2008;6:505-13; quiz 14.

11. Roth P, Happold C, Weller M. Corticosteroid use in neurooncology: an update. Neurooncol Pract 2015;2:6-12.

12. Mikkelsen T, Paleologos NA, Robinson PD, et al. The role of prophylactic anticonvulsants in the management of brain metastases: a systematic review and evidence-based clinical practice guideline. J Neurooncol 2010;96:97-102.

13. Young DF, Posner JB, Chu F, et al. Rapid-course radiation therapy of cerebral metastases: results and complications. Cancer 1974;34:1069-76.

14. Hindo WA, DeTrana FA, Lee MS, et al. Large dose increment irradiation in treatment of cerebral metastases. Cancer 1970;26:138-41.

15. Sharma A, Mountjoy LJ, Butterfield RJ, et al. Expanding the Spectrum of Radiation Necrosis After Stereotactic Radiosurgery (SRS) for Intracranial Metastases From Lung Cancer: A Retrospective Review. Am J Clin Oncol 2020;43:128-32.

16. Wang TJ, Saad S, Qureshi YH, et al. Does lung cancer mutation status and targeted therapy predict for outcomes and local control in the setting of brain metastases treated with radiation? Neuro Oncol 2015;17:1022-8.

17. Sharma M, Jia X, Ahluwalia M, et al. First followup radiographic response is one of the predictors of local tumor progression and radiation necrosis after stereotactic radiosurgery for brain metastases. Cancer Med 2017;6:2076-86.

18. Minniti G, Clarke E, Lanzetta G, et al. Stereotactic radiosurgery for brain metastases: analysis of outcome and risk of brain radionecrosis. Radiat Oncol 2011;6:48.

19. Boothe D, Young R, Yamada Y, et al. Bevacizumab as a treatment for radiation necrosis of brain metastases post stereotactic radiosurgery. Neuro Oncol 2013;15:1257-63.

20. Delishaj D, Ursino S, Pasqualetti F, et al. Bevacizumab for the Treatment of Radiation-Induced Cerebral Necrosis:
A Systematic Review of the Literature. J Clin Med Res 2017;9:273-80.

21. Dye NB, Gondi V, Mehta MP. Strategies for preservation of memory function in patients with brain metastases. Chin Clin Oncol 2015;4:24.

22. Day J, Zienius K, Gehring K, et al. Interventions for preventing and ameliorating cognitive deficits in adults treated with cranial irradiation. Cochrane Database Syst Rev 2014;2014:CD011335.

23. Brown PD, Pugh S, Laack NN, et al. Memantine for the prevention of cognitive dysfunction in patients receiving whole-brain radiotherapy: a randomized, double-blind, placebo-controlled trial. Neuro Oncol 2013;15:1429-37.

24. Brown PD, Gondi V, Pugh S, et al. Hippocampal Avoidance During Whole-Brain Radiotherapy Plus Memantine for Patients With Brain Metastases: Phase III Trial NRG Oncology CC001. J Clin Oncol 2020;38:1019-29.

25. Rittmeyer A, Barlesi F, Waterkamp D, et al. Atezolizumab versus docetaxel in patients with previously treated non-small-cell lung cancer (OAK): a phase 3, openlabel, multicentre randomised controlled trial. Lancet 2017;389:255-65.

26. Shah M, Tayar JH, Abdel-Wahab N, et al. Myositis as an adverse event of immune checkpoint blockade for cancer therapy. Semin Arthritis Rheum 2019;48:736-40.

27. Okada H, Weller M, Huang R, et al. Immunotherapy response assessment in neuro-oncology: a report of the RANO working group. Lancet Oncol 2015;16:e534-e542.

28. Temel JS, Greer JA, Muzikansky A, et al. Early palliative care for patients with metastatic non-small-cell lung cancer. N Engl J Med 2010;363:733-42.

29. Temel JS, Greer JA, El-Jawahri A, et al. Effects of Early Integrated Palliative Care in Patients With Lung and GI Cancer: A Randomized Clinical Trial. J Clin Oncol 2017;35:834-41.

30. Koekkoek JAF, Dirven L, Reijneveld JC, et al. Epilepsy in the end of life phase of brain tumor patients: a systematic review. Neuro-Oncology Practice 2014;1:134-40.

31. Orrevall Y. Nutritional support at the end of life. Nutrition 2015;31:615-6.

Cite this article as: Sharma A, Mrugala MM. Supportive care for patients with brain metastases from lung cancer. J Thorac Dis 2021;13(5):3258-3268. doi: 10.21037/jtd-2019-rbmlc-11 